\title{
ON THE ERGODIC THEOREM
}

\author{
BY \\ NELSON DUNFORD AND D. S. MILLER
}

Introduction. The purpose of this note is to show that for transformations in a Lebesgue space $L(S)$ (where $S$ has finite measure) of the type $T f=f(\phi t)$ (where $\phi$ is a map of $S$ into all or part of itself, not necessarily one-to-one and not necessarily measure preserving) the pointwise convergence of

$$
T_{n}(f, t)=\frac{1}{n} \sum_{n=0}^{n-1} f\left(\phi^{\nu} t\right)
$$

is a consequence of its mean convergence and that its mean convergence is equivalent to the statement

$$
\sum_{v=0}^{n-1}\left|\phi^{-v} e\right| \leqq K|e|,
$$$$
n=1,2, \cdots,
$$

where $K$ is independent of the measurable set $e$ in $S$ and $\phi^{-v} e$ is the set of those $t \in S$ for which $\phi^{\star} t \in e$. We have also proved an analogous theorem for a measurable $n$-parameter semi-group of transformations in $S$. This result, for $n=1$, is as follows: the pointwise convergence (almost everywhere) of

$$
\frac{1}{\gamma} \int_{0}^{\gamma} f\left(\phi^{\lambda} t\right) d \lambda
$$

is a consequence of its mean convergence which is equivalent to the statement

$$
\frac{1}{\gamma} \int_{0}^{\gamma}\left|\phi^{-\lambda} e\right| \leqq M_{\gamma}|e|, \quad \quad \limsup _{\gamma \rightarrow \infty} M_{\gamma}<\infty .
$$

Our methods are closely related to a combination of those of F. Riesz $\left.{ }^{1}\right)$, K. Yosida $\left({ }^{2}\right)$, S. Kakutani $\left({ }^{3}\right)$, and H. R. Pitt $\left({ }^{4}\right)$. The basic principle used to get the pointwise convergence is due to $\mathrm{S}$. Banach $\left({ }^{5}\right)$.

The discrete case. Let $|e|$ be a countably additive measure defined for

Presented to the Society, September 13, 1943; received by the editors April 23, 1945.

(1) F. Riesz, Some mean ergodic theorems, J. London Math. Soc. vol. 13 (1938) pp. 274-278.

(2) K. Yosida, An abstract treatment of the individual ergodic theorem, Proc. Imp. Acad. Tokyo vol. 16 (1940) pp. 280-284.

(3) S. Kakutani, Mean ergodic theorem in abstract (L)-spaces, Proc. Imp. Acad. Tokyo vol. 15 (1939) pp. 121-123.

(4) H. R. Pitt, Some generalizations of the ergodic theorem, Proc. Cambridge Philos. Soc. vol. 38 (1942) pp. 325-343.

(5) S. Banach, Sur la convergence presque partout des fonctionnelles linéaires, Bull. Sci. Math. vol. 50 (1926) pp. 36-43. 
the sets $e$ in a Borel field $\mathcal{F}$ of subsets of a set $S$. We suppose that $S \in \mathcal{F}$ and $|S|<\infty$. The symbol $L(S)$ will be used for the Banach space of real summable functions on $S$. The symbol $\phi$ will be used for a mapping of $S$ into itself and we shall assume

(1) $\phi S \subset S$,

(2) $\phi^{-1} e \in \mathcal{F}$ if $e \in \mathcal{F}$,

(3) $\left|\phi^{-1} e\right|=0$ if $|e|=0$.

Here the symbol $\phi^{-1} e$ stands for the set of all $t \in S$ for which $\phi t \in e$. It is not assumed that $\phi$ is one-to-one. Let $T$ be a transformation in the linear class of real functions on $S$ defined by $T f=g, g(t)=f(\phi t)$. In general when dealing with transformations on function classes we shall find it convenient to use the symbol $T(f, t)$ for the value of $T f$ at the point $t$. Thus $T(f, t)=f(\phi t)$.

THEOREM 1. The first two of the following statements are equivalent and either one implies the third.

(4) There is a constant $M$ such that

$$
\frac{1}{n} \sum_{v=0}^{n-1}\left|\phi^{-v} e\right| \leqq M|e|, \quad e \in \mathcal{F}, n=1,2, \cdots .
$$

(5) For every $f \in L(S)$ we have $T f \in L(S)$ and

$$
\frac{1}{n} \sum_{\nu=0}^{n-1} T^{\nu} f \text { converges in } L(S) \text {. }
$$

(6) For every $f$ in $L(S)$ the limit

exists for almost all $t \in S$.

$$
\lim _{n \rightarrow \infty} \frac{1}{n} \sum_{\nu=0}^{n-1} f\left(\phi^{\nu} t\right)
$$

LEMMA 1. The following three statements are equivalent.

(a) If $f \in L(S)$ then $T f \in L(S)$.

(b) $T$ is a continuous linear operator in $L(S)$.

(c) There is a constant $K$ such that $\left|\phi^{-1} e\right| \leqq K|e|, e \in \mathcal{F}$.

To see that (a) implies (b) it will suffice to prove that $T$ is closed $\left(^{(}\right)$. Let $f^{n} \rightarrow f, T f^{n} \rightarrow g$ in $L(S)$. There is a subsequence $f^{n_{i}}$ with $f^{n_{i}}(t) \rightarrow f(t), T\left(f^{n_{i}}, t\right)$ $=f^{n_{i}}(\phi t) \rightarrow g(t)$ almost everywhere in $S$. Since $\left|\phi^{-1} e\right|=0$ if $|e|=0$ (3) we have $f^{n_{i}}(\phi t) \rightarrow f(\phi t)$ for almost all $t$. Thus $T f=g$ in $L(S)$ and so $T$ is closed and hence continuous. If $T$ is continuous there is a constant $K$ such that $|T f| \leqq K|f|$. If we let $f$ be the characteristic function of a set $e \in \mathcal{F}$ the preceding inequality yields (c). Hence (b) implies (c). Assuming (c) and letting $f(t)=\sum \alpha_{i} \psi\left(e_{i}, t\right)$ where $e_{i}$ are disjoint sets in $\mathcal{F}$ and $\psi(e)$ is the characteristic function of $e$, we have

(') S. Banach, Theorie des opérations linéaires, Warsaw, 1932, p. 41. 


$$
|T f|=\int_{S}|f(\phi t)| d t \leqq \sum\left|\alpha_{i}\right|\left|\phi^{-1} e_{i}\right| \leqq K|f| .
$$

Thus $T$ is continuous on a dense subset of $L(S)$. Let $T$ be the unique continuous extension of $T$ to the whole space $L(S)$. Choose a sequence $f^{n}$ of step functions (finitely-valued measurable functions) such that $f^{n} \rightarrow f$ in $L(S), f^{n}(t)$ $\rightarrow f(t), \tilde{T}\left(f^{n}, t\right) \rightarrow \tilde{T}(f, t)$ for almost all $t \in S$. Clearly $\tilde{T}\left(f^{n}, t\right)=f^{n}(\phi t) \rightarrow f(\phi t)$ for almost all $t$ and so $\tilde{T}(f, t)=f(\phi t)$ almost everywhere. This proves that $T f \in L(S)$ and shows that (c) implies (a).

Occasionally in what follows we shall write $T_{n} f$ for $(1 / n) \sum_{v=0}^{n-1} T^{\prime} f$. We shall always use the symbol $\psi(e)$ for the characteristic function of $e$.

LEMMA 2. The transformation $T$ is a continuous linear operator in $L(S)$ with $\left|T_{n}\right|$ bounded if and only if (4) holds.

Suppose that $T$ is a continuous linear operator in $L(S)$ with $\left|T_{n} f\right| \leqq M|f|$, $f \in L(S)$. By placing $f=\psi(e)$ in this inequality we obtain (4). Conversely using (4), first with $n=2$, we find that $\left|\phi^{-1} e\right| \leqq(2 M-1)|e|$ which shows in view of Lemma 1 that $T$ is a continuous linear operator in $L(S)$. If $f$ is a step function defined by the equation $f(t)=\sum \alpha_{i} \psi\left(e_{i}, t\right)$, with $e_{i}$ disjoint, we have

$$
\begin{aligned}
\left|T_{n} f\right| & \leqq \sum\left|\alpha_{i}\right| \frac{1}{n} \sum_{v=0}^{n-1} \int_{S} \psi\left(e_{i}, \phi^{\nu} t\right) d t \\
& =\sum\left|\alpha_{i}\right| \frac{1}{n} \sum_{v=0}^{n-1}\left|\phi^{->} e_{i}\right| \leqq M|f| .
\end{aligned}
$$

Since $T_{n}$ is continuous in $L(S),\left|T_{n}\right| \leqq M$.

A set $L_{0} \subset L(S)$ is said to be weakly compact if for every sequence $f^{n} \in L_{0}$ there is a subsequence $f^{n i}$ and an $f \in L(S)$ such that $x^{*} f^{n i} \rightarrow x^{*} f$ for every linear functional $x^{*}$ defined on $L(S)$.

LEMMA 3. A set $L_{0} \subset L(S)$ is weakly compact if and only if there is a constant $K$ such that $|f| \leqq K, f \in L_{0}$, and

$$
\lim _{|\ell| \rightarrow 0} \int_{0} f(t) d t=0 \text { uniformly for } f \in L_{0} .
$$

The proof of this lemma has been given elsewhere $\left({ }^{7}\right)$.

LEMma 4. Statements (4) and (5) are equivalent.

Assuming (5) we see first by Lemma 1 that $T$ is a continuous operator in $L(S)$. The convergence of $T_{n}$ implies (using the principle of uniform boundedness $\left({ }^{8}\right)$ ) that $\left|T_{n}\right|$ is bounded. Hence from Lemma 2 we conclude that (5)

(7) N. Dunford, A mean ergodic theorem, Duke Math. J. vol. 5 (1939) pp. 635-646.

(8) S. Banach, loc. cit. p. 80. 
implies (4). We now assume (4) and let $L_{0}\left(L_{1}\right)$ be the set of $f \in L(S)$ for which the sequence $\left\{T_{n} f\right\}$ is weakly compact (convergent). It is clear that $L_{0}, L_{1}$ are linear manifolds in $L(S)$ with $L_{1} \subset L_{0}$ and that (since (4) implies the boundedness of $\left.\left|T_{n}\right|\right) L_{1}$ is closed. It is also clear from Lemma 3 that every bounded function in $L(S)$ belongs to $L_{0}$ and hence $L_{0}$ is dense in $L(S)$. Thus to prove (5) it will suffice to show that $L_{0} \subset L_{1}$. Let $\mathfrak{M}$ be the set of $g \in L(S)$ for which $T g=g$ and $\mathfrak{R}=(I-T) L(S)$. Since $T_{n} g=g$ for $g \in \mathfrak{M},\left|T_{n}\right|$ is bounded (Lemma 2), and $T_{n}(I-T) f=(1 / n)\left(I-T^{n}\right) f \rightarrow 0$, we see that $c(\mathfrak{R}) \mathfrak{M}=0$ and $\mathfrak{M} \oplus c(\mathfrak{R}) \subset L_{1}$ (where $c(\mathfrak{R})$ is the closure of $\left.\mathfrak{R}\right)$. Now let $f \in L_{0}$ and choose $n_{i}$ and $g \in L(S)$ so that $T_{n_{i}} f \rightarrow g$ weakly. Since $(I-T) T_{n_{i}} f \rightarrow 0$ we have $g=T g=T_{n} g$ $\in \mathbb{M}$. The vector $h=f-g$ is in $c(\mathfrak{N})$. To see this we suppose the contrary and pick a functional $x^{*}$ with $x^{*} h=1, x^{*} \mathfrak{R}=0$. Since $\left(I-T^{n}\right) h=(I-T) h$ $+(I-T) T h+\cdots+(I-T) T^{n-1} h \in \mathfrak{R}$ we see that $x^{*} h=x^{*} T^{n} h=x^{*} T_{n} h$, $n=1,2, \cdots$, and hence that $1=x^{*} h=x^{*} T_{n_{i}} h=x^{*} T_{n_{i}} f-x^{*} g \rightarrow 0$, a contradiction. Thus we may say that an arbitrary $f \in L_{0}$ is of the form $g+h$ where $g \in \mathfrak{M}, h \in c(\mathfrak{N})$. Since $\mathfrak{M} \oplus c(\mathfrak{N}) \subset L_{1}$ we have proved that $L_{0} \subset L_{1}$ and completed the proof of the lemma. During the course of this proof we have established the fact that if (4) holds then

(7) $\mathfrak{M} \oplus c(\mathfrak{R})=L(S)$

which will be needed later.

LEMMA 5. Let $\alpha>0, m \geqq 1, r_{n} \geqq \alpha, n=0,1, \cdots$, and

$$
\operatorname{loc}_{0<p \geqq m} \frac{1}{p} \sum_{p=0}^{p-1} r_{p+n} \geqq \alpha, \quad n=0,1,2, \cdots \text {. }
$$

Then

$$
\liminf _{n \rightarrow \infty} \frac{1}{n} \sum_{r=0}^{n-1} r_{\nu} \geqq \alpha .
$$

Fix $n>m, n_{0}=0$, and $p_{1}$ such that $0<p_{1} \leqq m$ and $\sum_{v=0}^{p_{1}-1} r_{v+n_{0}} \geqq \alpha p_{1}$. Let $n_{1}=p_{1}$ and choose $p_{2}$ such that $0<p_{2} \leqq m$ and $\sum_{\nu=0}^{p_{2}-1} r_{\nu+n_{1}} \geqq \alpha p_{2}$. Let $n_{2}=p_{1}+p_{2}$ and choose $p_{3}$ such that $0<p_{3} \leqq m$ and $\sum_{\nu=0}^{p_{2}-1} r_{v+n_{3}} \geqq \alpha p_{3}$. Continuing in this way we arrive at an integer $q$ such that $n-m \leqq n_{q} \leqq n, n_{q}=\sum_{i=1}^{q} p_{i}, 0<p_{i} \leqq m$, $\sum_{v=0}^{p_{i}-1} r_{v+n_{i-1}} \geqq \alpha p_{i}(i=1, \cdots, q)$. Thus

$$
\frac{1}{n} \sum_{v=0}^{n-1} r_{\nu} \geqq \frac{1}{n} \sum_{\nu=0}^{n_{4}-1} r_{\nu} \geqq \frac{\alpha n_{q}}{n} \geqq \alpha\left(1-\frac{m}{n}\right) .
$$

which proves the lemma.

For the statement of the next lemma it will be convenient to introduce the following notation: $f_{n}=T_{n} f, \bar{f}_{m}(t)=$ l.u.b. $0 \leq_{n \leq m} f_{n}(t), \bar{f}(t)=1$.u.b. $0 \leq n<\infty f_{n}(t)$, $e_{\alpha}^{n}=\varepsilon_{t}\left[\bar{f}_{n}(t)>\alpha\right], \quad e_{\alpha}=\lim _{n \rightarrow \infty} e_{\alpha}^{n}, \quad e=\varepsilon_{t}[\bar{f}(t)=\infty]$. Clearly $\bar{f}_{n}(t) \uparrow f(t), \quad e_{\alpha}^{n} \uparrow e_{\alpha}$ $=\varepsilon_{t}[\bar{f}(t)>\alpha]$, and $e_{\alpha} \downarrow e$.

Lemma 6. If (4) holds then for every $f \in L(S)$ the corresponding set e has measure zero. 
We may and shall assume that $f(t) \geqq 0$. Fix the integer $m \geqq 1$ and the positive number $\alpha$. Define

$$
g(t)=\psi\left(e_{\alpha}^{m}, t\right) f(t)+\alpha \psi\left(\widetilde{e}_{\alpha}^{m}, t\right),
$$

where $\psi(e)$ is the characteristic function of the set $e$. In view of Lemma 4 there is a continuous linear operator $E$ in $L(S)$ with $T_{n} h \rightarrow E h, h \in L(S)$. Note that if $0 \leqq h(t) \leqq f(t)$ then $0 \leqq h_{n}(t) \leqq f_{n}(t)$ and $0 \leqq E(h, t) \leqq E(f, t)$ for almost all $t \in S$, and hence $|E h| \leqq|E f|$. This fact together with $\left(^{*}\right)$ gives

$$
|E g| \leqq|E f|+\alpha\left|E \psi\left(\widetilde{e}_{\alpha}^{m}\right)\right| \text {. }
$$

Now $f(t) \leqq \bar{f}_{m}(t)$ from which it follows that $f(t) \leqq g(t)$ and hence that $\bar{f}_{m}(t)$ $\leqq \bar{g}_{m}(t)$. If for some integer $n$ and $t \in S$ we have $\phi^{n} t \in e_{\alpha}^{m}$, then $\bar{g}_{m}\left(\phi^{n} t\right) \geqq \bar{f}_{m}\left(\phi^{n} t\right)$ $>\alpha$. On the other hand if $\phi^{n} t \in \widetilde{e}_{\alpha}^{m}$ then $\bar{g}_{m}\left(\phi^{n} t\right) \geqq g\left(\phi^{n} t\right)=\alpha$ so that $\bar{g}_{m}\left(\phi^{n} t\right) \geqq \alpha$, $t \in S, n=0,1, \cdots$. We may thus apply Lemma 5 with $r_{n}=g\left(\phi^{n} t\right)$ to see that $\lim _{\inf } f_{n \rightarrow \infty} g_{n}(t) \leqq \alpha$. It follows at once from $\left({ }^{* *}\right)$ and Fatou's lemma that

$$
\alpha|S| \leqq \lim _{n \rightarrow \infty} \int_{S} g_{n}(t) d t=|E g| \leqq|E f|+\alpha\left|E \psi\left(\tilde{e}_{\alpha}^{m}\right)\right| .
$$

Since $\psi\left(\tilde{e}_{\alpha}^{m}\right) \rightarrow \psi\left(\tilde{e}_{\alpha}\right)$ and $E$ is continuous we see from the above inequality, by letting $m \rightarrow \infty$, that $|S| \leqq \alpha^{-1}|E f|+\left|E \psi\left(\tilde{e}_{\alpha}\right)\right|$. By letting $\alpha \rightarrow \infty$ we obtain $|S| \leqq|E \psi(\widetilde{e})|$. Since $0 \leqq \psi(\widetilde{e}, t) \leqq 1$ we have $0 \leqq E(\psi(\widetilde{e}), t) \leqq 1$ almost everywhere and hence $|E \psi(\tilde{e})| \leqq|S|$ which proves that $|E \psi(\tilde{e})|=|S|$. Thus $E(\psi(\widetilde{e}), t)=1$ almost everywhere on $S$. Now $1=E(1)=E(\psi(\widetilde{e}), t)+E(\psi(e), t)$ almost everywhere and hence $E(\psi(e), t)=0$ almost everywhere, that is, $E \psi(e)=0$. Now since

$$
f_{n}(\phi t)=\frac{n+1}{n} f_{n+1}(t)-\frac{1}{n} f(t)
$$

we see that $t \in e$ if and only if $\phi t \in e$. This gives $e \subset \phi^{-1} e \subset e$ and hence $e=\phi^{-1} e$ $=\phi^{-v} e, \nu=1,2, \cdots$. Thus we have

$$
0=|E \psi(e)|=\lim _{n \rightarrow \infty} \int_{S} \frac{1}{n} \sum_{v=0}^{n-1} \psi(e)\left(\phi^{n} t\right) d t=\lim _{n \rightarrow \infty} \frac{1}{n} \sum_{n=0}^{n-1}\left|\phi^{-v} e\right|=|e|,
$$

which completes the proof of Lemma 6 .

Let $M(S)$ be the complete linear metric space of real finite measurable functions on $S$ with $(f, g)=|f-g|$ where $|f|=\int_{S}|f(t)| d t /(1+|f(t)|)$.

LEMma 7. Let $\Lambda_{1} \supset \Lambda_{2} \supset \ldots$ be denumerable sets of elements and for each $\gamma \in \Lambda_{1}$ let $T_{\gamma}$ be a continuous linear transformation from $L(S)$ to $M(S)$. If

(a) for $f \in L(S)$, l.u.b.r $\in \Lambda_{1}\left|T_{\gamma}(f, t)\right|<\infty$, for almost all $t \in S$, and

(b) for each $f$ in a dense set in $L(S)$ 
then

$$
\lim _{n} \sup _{\gamma \in \Lambda_{n}} T_{\gamma}(f, t)=\lim _{n} \inf _{\gamma \in \Lambda_{n}} T_{\gamma}(f, t), \text { for almost all } t \in S
$$

(c) the equation appearing in (b) holds for every $f \in L(S)$.

This lemma is due essentially to S. Banach $\left({ }^{\circ}\right)$. The proof given here is due to S. Mazur and W. Orlicz $\left({ }^{10}\right)$ and the formulation which is a bit more complicated than that of Mazur and Orlicz is a modification of one suggested by Federer. The more complicated formulation is not necessary for the discrete case of the ergodic theorem discussed here but will be convenient in the discussion of the case of a flow dependent on a continuous parameter.

Let $\gamma_{1}, \gamma_{2}, \cdots$ be an enumeration of the elements in $\Lambda_{1}$. Let $V_{n}(f, t)$

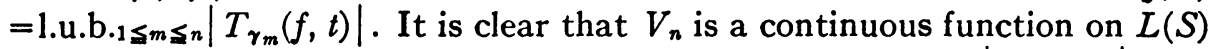
to $M(S)$ and that $V_{n} f \rightarrow V f, f \in L(S)$, where $V(f, t)=$ l.u.b.r $\in_{\Lambda_{1}}\left|T_{\gamma}(f, t)\right|$. Let $L_{k}, k=1,2, \cdots$, be the set of $f \in L(S)$ for which $\left|(1 / k) V_{n} f\right| \leqq \epsilon / 2$, $n=1,2, \cdots$. Since $V_{n} f$ converges for every $f \in L(S)$ we have $L(S)=\sum L_{k}$. Since the functions $V_{n}$ are continuous the set $L_{k}$ is closed and hence by the Baire category theorem there is a $k_{0}$ and a sphere with center $f_{0}$ and radius $r>0$ which is contained in $L_{k_{0}}$. This means that

and hence

$$
\left|\frac{1}{k_{0}} V_{n}\left(f_{0}+f\right)\right| \leqq \frac{\epsilon}{2}, \quad n=1,2, \cdots ;|f| \leqq r,
$$

$$
\left|\frac{1}{k_{0}} V_{n} f\right| \leqq\left|\frac{1}{k_{0}} V_{n}\left(f_{0}+f\right)\right|+\left|\frac{1}{k_{0}} V_{n} f_{0}\right| \leqq \epsilon, \quad|f| \leqq r ; n=1,2, \cdots .
$$

Thus if $\delta=r / k_{0}$ we have

$$
\left|V_{n} f\right|=\left|\frac{1}{k_{0}} V_{n} k_{0} f\right| \leqq \epsilon, \quad n=1,2, \cdots ;|f| \leqq \delta,
$$

and $|V f| \leqq \epsilon,|f| \leqq \delta$. Let $W$ be defined on $L(S)$ to $M(S)$ by the equation

$$
W(f, t)=\lim _{n} \sup _{\gamma \in \Delta_{n}} T_{\gamma}(f, t)-\lim _{n} \inf _{\gamma \in \Delta_{n}} T_{\gamma}(f, t) ;
$$

then $|W(f, t)| \leqq 2 V(f, t)$ and hence $|W f| \leqq 2|V f| \leqq 2 \epsilon,|V f| \leqq \delta$. Thisshowsthat $W$ is continuous at the origin. But it is easily verified that $|W(f, t)-W(h, t)|$ $\leqq W(f-h, t)$ and hence $|W f-W h| \leqq|W(f-h)|$, which shows that $W$ is everywhere continuous. By the hypothesis (b), $W f=0$ for $f$ in a dense set in $L(S)$ and so $W f=0$ for all $f$ in $L(S)$, which proves the lemma.

LeMma 8. Let $T_{i}, i=1,2, \cdots$, be a continuous linear operator on $L(S)$ to $M(S)$. If

(?) See footnote 5 .

(10) S. Mazur and W. Orlicz, Über Folgen linearer Operationen, Studia Mathematica vol. 4 (1933) pp. 152-157. 
(a) for $f \in L(S)$, l.u.b. $1 \leq i<\infty|T,(f, t)|<\infty$ for almost all $t \in S$, and

(b) for $f$ in a dense subset of $L(S)$ the $\lim _{i \rightarrow \infty} T_{i}(f, t)$ exists almost everywhere on $S$, then

(c) for every $f \in L(S)$ the $\lim _{i \rightarrow \infty} T_{i}(f, t)$ exists almost everywhere on $S$.

This is a corollary of Lemma 7 and may be proved by taking $\Lambda_{n}$ in Lemma 7 to be the set of all integers $i \geqq n$.

\section{LeMma 9. Statement (4) implies statement (6).}

By Lemma 6, 1.u.b. $\left|T_{n}(f, t)\right|<\infty$ for almost all $t$ and hence for every $f \in L(S)$ the sequence $f\left(\phi^{n} t\right) / n=f(t) / n-(I-T) T_{n}(f, t)$ is bounded almost everywhere. On the other hand, $f\left(\phi^{n} t\right) / n \rightarrow 0$ if $f$ is a bounded function. Thus Lemma 8 shows that $T_{n}(h, t)$ converges almost everywhere for every $h$ of the form $(I-T) f$, that is, for every $h \in \mathfrak{R}$. Clearly $T_{n}(g, t)=g(t)$ converges almost everywhere for every $g \in \mathbb{M}$, that is, every $g$ for which $T g=g$. Thus $T_{n}(f, t)$ converges almost everywhere for $f \in \mathfrak{M}+\mathfrak{N}$. Such $f$ are by equation (7) dense in $L(S)$ and hence the desired conclusion follows from Lemma 6 and Lemma 7.

This completes the proof of Theorem 1.

The case of a continuous flow. Here $R$ will stand for those points $\left(\lambda_{1}, \cdots, \lambda_{n}\right)$ in Euclidean $n$-space for which $\lambda_{i} \geqq 0, i=1, \cdots, n$, and for brevity we write $\lambda$ for $\left(\lambda_{1}, \cdots, \lambda_{n}\right)$. For each $\lambda \in R$ there is a transformation $\phi^{\lambda}$ on $S$ which satisfies (1), (2), (3), and

$$
\phi^{\lambda+\mu t}=\phi^{\lambda} \phi^{\mu} t, \quad \phi^{0} t=t, \quad t \in S ; \lambda, \mu \in R,
$$

(9) for every $e \in \mathcal{F}$ the set of points $(\lambda, t) \in R \times S$ for which $\phi^{\lambda} t \in e$ is a measurable subset of $R \times S$.

(10) If the dimension $n \geqq 2$ it is assumed that $\phi^{\lambda} e \subset e, \lambda \in R$, for every set $e \in \mathcal{F}$ with the property that $\phi^{\lambda} \tilde{E} \subset \tilde{e}, \lambda \in R$.

The postulate (9) will assure (Lemma 10) the $(\lambda, t)$ measurability of $f\left(\phi^{\lambda} t\right)$ for every $f \in L(S)$ and in particular the $\lambda$ measurability of $\left|\phi^{-\lambda} e\right|$ for $e \in \mathcal{F}$. Thus all integrals appearing in the theorem to follow are Lebesgue integrals. We shall use the symbol $C(\gamma)$ for the cube composed of those $\lambda \in R$ for which $0 \leqq \lambda_{i} \leqq \gamma, i=1, \cdots, n$, the symbol $T_{\gamma}(f, t)$ for $\left(1 / \gamma^{n}\right) \int_{c(\gamma)} f\left(\phi^{\lambda} t\right) d \lambda$, and $T_{\gamma} f$ for the function $T_{\gamma}(f, \cdot)$.

THEOREM 2. The first two of the following statements are equivalent and either one implies the third. and

(11) For $\gamma>0$ there is a constant $M_{\gamma}$ with $\lim \sup _{\gamma \rightarrow \infty} M_{\gamma}<\infty$

$$
\frac{1}{\gamma^{n}} \int_{C(\gamma)}\left|\phi^{-\lambda} e\right| d \lambda \leqq M_{\gamma}|e|, \quad \gamma>0, e \in \mathcal{F}
$$


(12) If $f \in L(S)$ then $T_{\gamma} f \in L(S), \gamma>0$, and the $\lim _{\gamma \rightarrow \infty} T_{\gamma}$ exists in $L(S)$.

(13) For every $f \in L(S)$ the $\lim _{\gamma \rightarrow \infty} T_{\gamma}(f, t)$ exists for almost all $t \in S$.

LEMMA 10. For every $f \in L(S)$ the function $f\left(\phi^{\lambda} t\right)$ is measurable in $(\lambda, t)$ on the product space $R \times S$.

Statement (9) proves the lemma in case $f$ is the characteristic function of a set $e \in \mathcal{F}$. Thus the lemma is valid for step functions. Now for an arbitrary non-negative function $f \in L(S)$ we may choose a nondecreasing sequence $f_{\text {n }}$ of step functions with $f_{n}(t) \rightarrow f(t), t \in S$. Since

$$
\underset{(\lambda, t)}{\varepsilon}\left[f\left(\phi^{\lambda} t\right)>\alpha\right]=\sum_{1}^{\infty} \underset{(\lambda, t)}{\varepsilon}\left[f_{n}\left(\phi^{\lambda} t\right)>\alpha\right]
$$

we see that $f\left(\phi^{\lambda} t\right)$ is measurable on $R \times S$.

LemMa 11. For every $\gamma>0, T_{\gamma}$ is a continuous linear operator in $L(S)$ with $\lim \sup _{\gamma \rightarrow \infty}\left|T_{\gamma}\right|<\infty$ if and only if (11) holds.

If $T_{\gamma}$ has the required properties, (11) follows from the inequality $\left|T_{\gamma} f\right| \leqq\left|T_{\gamma}\right||f|$ by placing $f=\psi(e)$, the characteristic function of $e$, and applying the Fubini theorem. Conversely assume (11) and let $f(t)=\sum \alpha_{i} \psi\left(e_{i}, t\right)$. Then

$$
\left|T_{\gamma} f\right|=\int_{S}\left|T_{\gamma}(f, t)\right| d t \leqq \sum\left|\alpha_{i}\right| \frac{1}{\gamma^{n}} \int_{C(\gamma)}\left|\phi^{-\lambda} e_{i}\right| d \lambda \leqq M_{\gamma}|f| .
$$

For an arbitrary non-negative $f \in L(S)$ choose a nondecreasing sequence of step functions $f_{n}$ with $f_{n}(t) \rightarrow f(t), t \in S$. Then by Lebesgue's theorem for monotone sequences we have

$$
\left|T_{\gamma} f_{n}\right| \rightarrow \int_{S} d t \frac{1}{\gamma^{n}} \int_{C(\gamma)} f\left(\phi^{\lambda} t\right) d \lambda
$$

and since $\left|T_{\gamma} f_{n}\right| \leqq M_{\gamma}\left|f_{n}\right| \rightarrow M_{\gamma}|f|$, we have the desired result. The above argument shows that $\int_{S} d t \int_{C(\gamma)}\left|f\left(\phi^{\lambda} t\right)\right| d \lambda$ exists finite for every $\gamma>0$ and every $f \in L(S)$. Thus by the Fubini theorem we have the following lemma.

LEMMA 12. If (11) holds then for every $f \in L(S)$ the function $f\left(\phi^{\lambda} t\right)$ is integrable over $S \times C(\gamma), \gamma>0$, and $f\left(\phi^{\lambda} t\right)$ is integrable in $\lambda$ on $C(\gamma), \gamma>0$, for almost all $t \in S$.

LEMMA 13. If (11) holds then $\lim _{\gamma \rightarrow \infty} T_{\gamma}\left(I-T_{\gamma_{0}}\right) f=0$ for every $\gamma_{0}>0$ and every $f \in L(S)$.

In view of Lemma 11 we have $\lim \sup _{\gamma \rightarrow \infty}\left|T_{\gamma}\left(I-T_{\gamma_{0}}\right)\right|<\infty$ and so it will be sufficient to prove that $T_{\gamma}\left(I-T_{\gamma_{0}}\right) f \rightarrow 0$ if $f$ is a non-negative bounded function, say $0 \leqq f(t) \leqq K, t \in S$. Let $h(t)=f(t)-T_{\gamma_{0}}(f, t)$, then 


$$
\begin{aligned}
T_{\gamma}(h, t) & =\frac{1}{\left(\gamma \gamma_{0}\right)^{n}} \int_{C\left(\gamma_{0}\right)} d \lambda \int_{C(\gamma)}\left[f\left(\phi^{\nu} t\right)-f\left(\phi^{\lambda+\nu} t\right)\right] d \nu \\
& =\frac{1}{\left(\gamma \gamma_{0}\right)^{n}} \int_{C\left(\gamma_{0}\right)} d \lambda\left[\int_{A_{\lambda}} f\left(\phi^{\nu} t\right) d \nu-\int_{B_{\lambda}} f\left(\phi^{\nu} t\right) d \nu\right],
\end{aligned}
$$

where $A_{\lambda}=C(\gamma)-C(\gamma)(\lambda+C(\gamma)), \quad B_{\lambda}=(\lambda+C(\gamma))-C(\gamma)(\lambda+C(\gamma)), \quad$ and $\lambda \dot{+} C(\gamma)$ consists of all vectors of the form $\lambda+\mu$ where $\mu \in C(\gamma)$. The integrals $\int_{A_{\lambda}}, \int_{B_{\lambda}}$ appearing above are continuous positive functions of $\lambda$ on $C\left(\gamma_{0}\right)$ and will assume maximum values at certain points $\lambda_{1}, \lambda_{2}$ in $C\left(\gamma_{0}\right)\left(\lambda_{1}, \lambda_{2}\right.$ may of course depend upon $\gamma$ ). Thus

$$
\left|T_{\gamma}(h, t)\right| \leqq \frac{1}{\gamma^{n}} \int_{A_{\lambda_{1}}} f\left(\phi^{\nu} t\right) d \nu+\frac{1}{\gamma^{n}} \int_{B_{\lambda_{2}}} f\left(\phi^{\nu} t\right) d \nu,
$$

and so $\left|T_{\gamma} h\right| \leqq K|S|\left(\left|A_{\lambda_{1}}\right|+\left|B_{\lambda_{2}}\right|\right) / \gamma^{n} \rightarrow 0$.

\section{LEMMA 14. Statements (11) and (12) are equivalent.}

If (12) holds then by the principle of uniform boundedness we must have $\lim \sup _{\gamma \rightarrow \infty}\left|T_{\gamma}\right|<\infty$ and hence Lemma 11 gives (11). Conversely assume (11) or equivalently lim sup $\operatorname{su}_{\gamma \rightarrow \infty}\left|T_{\gamma}\right|<\infty$. Let $L_{0}$ consist of those $f \in L(S)$ for which the set $T_{\gamma_{i}} f$, for every sequence $\gamma_{i} \rightarrow \infty$, is weakly compact in $L(S)$. Just as before we see (Lemma 3) that the bounded functions in $L(S)$ are in $L_{0}$ and hence that $L_{0}$ is dense in $L(S)$. Let $L_{1}$ consist of those $f \in L(S)$ for which the $\lim _{\gamma \rightarrow \infty} T_{\gamma} f$ exists in $L(S)$. Since $\lim \sup _{\gamma \rightarrow \infty}\left|T_{\gamma}\right|<\infty$ we conclude that $L_{1}$ is a closed linear manifold in $L(S)$. Clearly $L_{1} \subset L_{0}$ and so to prove (12) it will be sufficient to show that $L_{0} \subset L_{1}$. Let $f \in L_{0}$ and choose $\gamma_{i} \rightarrow \infty, g \in L(S)$ such that $T_{\gamma_{i}} f \rightarrow g$ weakly. Let $\mathfrak{M}$ consist of those $g \in L(S)$ for which $T_{\gamma} g=g, \gamma>0$, and let $\mathfrak{N}$ be the linear manifold determined by all vectors of the form $\left(I-T_{\gamma}\right) f, \gamma>0, f \in L(S)$. Now $T_{\gamma} T_{\gamma_{i}} f \rightarrow T_{\gamma} g$ weakly and hence, by Lemma 13, $x^{*}\left(g-T_{\gamma} g\right)=\lim _{i} x^{*} T_{\gamma_{i}}\left(f-T_{\gamma} f\right)=0$, which shows that $g \in \mathfrak{M}$. The vector $h=f-g$ is in $c(\mathfrak{R})$, for if $x^{*}$ is any functional vanishing on $\mathfrak{R}$ we have $x^{*} f=x^{*} T_{\gamma} f, \gamma>0$, and hence $x^{*} f=x^{*} g, x^{*} h=0$. Thus we have shown that every $f \in L_{0}$ may be written as $f=g+h$ where $g \in \mathfrak{M}$ and $h \in c(\mathfrak{R})$. Clearly Lemma 13 together with the fact that $\lim \sup _{\gamma \rightarrow \infty}\left|T_{\gamma}\right|<\infty$ implies that $T_{\gamma} h \rightarrow 0$ for every $h \in c(\mathfrak{N})$ and so $L_{0} \subset L_{1}$ which proves the lemma. We have incidentally established the fact that if (11) holds then

(15) $L(S)=\mathfrak{M} \oplus c(\mathfrak{R})$.

LEMma 15. Let $\gamma_{\lambda}>0$ be defined for almost all $\lambda \in C\left(\gamma_{0}\right)$. Then among the cubes $\lambda+C\left(\gamma_{\lambda}\right)$ there are a finite number which are disjoint and whose union has measure exceeding $K_{n} \gamma_{0}^{n}$ where $K_{n}$ is an absolute constant depending only on the dimension $n$ of $R$. 
This is analogous to a covering theorem due to $\mathrm{N}$. Wiener( $\left.{ }^{11}\right)$ and the proof may be made by following Wiener's argument.

LemMa (12) 16. Let $\alpha>0, \beta>0, r(\lambda) \geqq 0$ and integrable on every $C(\gamma)$. Furthermore, for almost all $\lambda \in R$, let either

(i)

$$
r(\lambda) \geqq \alpha
$$

or

(ii)

$$
\underset{0<\gamma \geqq \beta}{\text { l.u.b. }} \frac{1}{\gamma^{n}} \int_{C(\gamma)} r(\lambda+\mu) d \mu \geqq \alpha \text {. }
$$

Then

$$
\liminf _{\gamma \rightarrow \infty} \frac{1}{\gamma^{n}} \int_{C(\gamma)} r(\lambda) d \lambda \geqq \alpha K_{n,}
$$

where $K_{n}$ is the absolute constant of Lemma 15 .

Since for almost all $\lambda \in R$,

$$
\lim _{\lambda \rightarrow 0} \frac{1}{\gamma^{n}} \int_{C(\gamma)} r(\lambda+\mu) d \mu=r(\lambda),
$$

we may assume that (ii) holds for almost all $\lambda \in R$ : Let $\epsilon>0$ and choose $\gamma>\beta$. Then for almost all $\lambda \in C(\gamma-\beta)$ there is a $\gamma_{\lambda}$ with $0<\gamma_{\lambda} \leqq \beta$ and

$$
\int_{C\left(\gamma_{\lambda}\right)} r(\lambda+\mu) d \mu \geqq(\alpha-\epsilon) \gamma_{\lambda}^{n} .
$$

By Lemma 15 there are points $\lambda_{i} \in C(\gamma-\beta) ; i=1, \cdots, k$, with $\left(\lambda_{i}+C\left(\gamma_{\lambda_{i}}\right)\right)$ $\cdot\left(\lambda_{j}+C\left(\gamma_{\lambda_{j}}\right)\right)=0, i \neq j$ and

Now

$$
\sum_{j=1}^{k}\left|C\left(\gamma_{\lambda_{j}}\right)\right|=\sum_{j=1}^{k} \gamma_{\lambda_{j}}^{n} \geqq K_{n}(\gamma-\beta)^{n} .
$$

$$
\begin{aligned}
\frac{1}{\gamma^{n}} \int_{C(\gamma)} r(\lambda) d \lambda & \geqq \frac{1}{\gamma^{n}} \sum_{j=1}^{k} \int_{\left.C . \gamma_{\lambda_{j}}\right)} r\left(\lambda_{j}+\mu\right) d \mu \\
& \geqq \frac{1}{\gamma^{n}} \sum_{j=1}^{k}(\alpha-\epsilon) \gamma_{\lambda_{j}}^{n} \geqq K_{n}(\alpha-\epsilon)(\gamma-\beta)^{n} / \gamma^{n} .
\end{aligned}
$$

By letting $\gamma \rightarrow \infty$ we obtain

$$
\liminf _{\gamma \rightarrow \infty} \frac{1}{\gamma^{n}} \int_{C(\gamma)} r(\lambda) d \lambda \geqq K_{n}(\alpha-\epsilon)
$$

(i1) N. Wiener, The ergodic theorem, Duke Math. J. vol. 5 (1939) pp. 1-18.

(12) This lemma was proved in the 1-dimensional case by H. R. Pitt, loc. cit. 
and since $\epsilon>0$ was arbitrary the proof is complete.

The next two lemmas will employ the following notation

$$
\begin{aligned}
& f_{\gamma}(t)=T_{\gamma}(f, t), \quad \bar{f}_{\beta}(t)=\text { l.u.b. }_{0<\gamma \leq \beta} f_{\gamma}(t), \quad \bar{f}(t)=\text { l.u.b. }_{0<\gamma<\infty} f_{\gamma}(t), \\
& e=\varepsilon_{t}[\vec{f}(t)=\infty], \quad e_{1}=\varepsilon\left[\limsup _{\gamma \rightarrow \infty} f_{\gamma}(t)=\infty\right], \\
& e_{\alpha}^{\beta}=\varepsilon_{t}\left[\bar{f}_{\beta}(t)>\alpha\right], \quad e_{\alpha}=\lim _{\beta \rightarrow \infty} e_{\alpha}^{\beta}
\end{aligned}
$$

Clearly $e=\lim _{\alpha \rightarrow \infty} e_{\alpha}$.

Lemma 17. Let $f \in L(S), f(t) \geqq 0$. Then $\phi^{\lambda} e_{1} \subset e_{1}, \phi^{-\lambda} e_{1} \subset e_{1}$ for $\lambda \in R$.

The proof of this lemma is the only place where (10) is used. Let $t \in \mathbb{e}_{1}$; then

$$
f_{\gamma}\left(\phi^{\mu} t\right)=\frac{1}{\gamma^{n}} \int_{\mu \dot{+} C(\gamma)} f\left(\phi^{\prime \prime} t\right) d \nu \leqq\left(1+\frac{|\mu|}{\gamma}\right)^{n} f_{\gamma+|\mu|}(t),
$$

where $|\mu|=\max _{1 \leq i \leq n}\left|\mu_{i}\right|$, and so $\phi^{\mu} \widetilde{e}_{1} \subset \widetilde{e}_{1}, \mu \in R$, which shows that $\phi^{-\mu} e_{1} \subset e_{1}$. Statement (10) gives (for $n \geqq 2) \phi^{\mu} e_{1} \subset e_{1}$. Now let $n=1$ and let $t \in e_{1}$. We have

$$
f_{\gamma}\left(\phi^{\mu} t\right)=f_{\gamma}(t)+\frac{1}{\gamma} \int_{\gamma}^{\gamma+\mu} f\left(\phi^{\nu} t\right) d \nu-\epsilon_{\gamma},
$$

where $\epsilon_{\gamma}=\gamma^{-1} \int_{0}^{\mu} f\left(\phi^{\nu} t\right) d \nu \rightarrow 0$ as $\gamma \rightarrow \infty$. Thus $\phi^{\mu} t \in e_{1}$ and $\phi^{\mu} e_{1} \subset e_{1}, \mu \in R$.

LEMMA 18. If (11) holds then for every $f \in L(S)$ the corresponding set $e_{1}$ has measure zero.

Let $E f$ be the point in $L(S)$ which in view of Lemma 14 exists and satisfies $T_{\gamma} f \rightarrow E f$. Clearly (Lemma 11$) E$ is a bounded linear operator in $L(S)$ with $|E| \leqq M$. We may and shall assume that $f(t) \geqq 0$. Fix $\alpha>0, \beta>0$ and define $g(t)=\psi\left(e_{\alpha}^{\beta}, t\right) f(t)+\alpha \psi\left(\tilde{e}_{\alpha}^{\beta}, t\right)$. Then $E g=E\left(\psi\left(e_{\alpha}^{\beta}\right) f\right)+\alpha E \psi\left(e_{\alpha}^{\beta}\right)$ and since $0 \leqq \psi\left(e_{\alpha}^{\beta}, t\right) f(t) \leqq f(t)$ we have $|E g| \leqq|E f|+\alpha\left|E \psi\left(\tilde{e}_{\alpha}^{\beta}, t\right)\right|$. By Lemma 12, for almost all $t \in S, f\left(\phi^{\lambda} t\right)$ is integrable in $\lambda$ on $C(\gamma)$ and hence for almost all $t \in S$

$$
\lim _{\gamma \rightarrow 0} \frac{1}{\gamma^{n}} \int_{C(\gamma)} f\left(\phi^{\lambda+\mu} t\right) d \mu=f\left(\phi^{\lambda} t\right)
$$

for almost all $\lambda$. Therefore for almost all $t \in S$ we have $f\left(\phi^{\lambda} t\right) \leqq \bar{f}_{\beta}\left(\phi^{\lambda} t\right)$ for almost all $\lambda$. From this fact and from the definition of $g$ we see that for almost all $t, f\left(\phi^{\lambda} t\right) \leqq g\left(\phi^{\lambda} t\right)$, for almost all $\lambda$. As a result of this inequality for almost all $t \in S$ either $\phi^{\lambda} t \in e_{\alpha}^{\beta}$ and $\bar{g}_{\beta}\left(\phi^{\lambda} t\right) \geqq \bar{f}_{\beta}\left(\phi^{\lambda} t\right) \geqq \alpha$ or $\phi^{\lambda} t \in \hat{e}_{\alpha}^{\beta}$ and $g\left(\phi^{\lambda} t\right)$ $=\alpha$. In Lemma 16 take $r(\lambda)=g\left(\phi^{\lambda} t\right)$ and by that lemma we conclude that for almost all $t \in S, \lim \inf _{\gamma \rightarrow \infty} g_{\gamma}(t) \geqq K_{n} \alpha$, and so by Fatou's lemma

$$
\alpha|S| K_{n} \leqq \lim _{\gamma}\left|g_{\gamma}\right|=|E g| \leqq|E f|+\alpha\left|E \psi\left(e_{\alpha}^{\beta}\right)\right| \text {. }
$$


Dividing by $\alpha$ and letting first $\beta \rightarrow \infty$ and then $\alpha \rightarrow \infty$ we get

$$
0<|S| K_{n} \leqq|E \psi(\widetilde{e})| \leqq M|\psi(\widetilde{e})|=M|\mathfrak{e}| \text {. }
$$

Thus the set $\tilde{e}$ of points $t \in S$ where $\vec{f}(t)<\infty$ has positive measure. Now this enables us to show that $\left|e_{1}\right|=0$. Suppose to the contrary that $\left|e_{1}\right|>0$. Since (Lemma 17) $\phi^{\lambda} e_{1} \subset e_{1}, \phi^{-\lambda} e_{1} \subset e_{1}, \lambda \in R$, we may apply the whole theory as developed for $S$ to $e_{1}$ and obtain from (16) the contradiction $0<\left|e_{1}\right| K_{n} \leqq M\left|e_{1} \varepsilon\right|$ $=0$.

LEMMA 19. If (11) holds then for every $f \in L(S)$ and $\gamma_{0} \in R$ and $h(t)=f(t)$ $-f_{\gamma_{0}}(t)$ we have $\lim _{\gamma \rightarrow \infty} h_{\gamma}(t)=0$ for almost all $t \in S$.

In view of Lemma 13 it will be sufficient to prove that $\lim _{\gamma} h_{\gamma}(t)$ exists almost everywhere. We have, from Lemma 18, that $\lim \sup _{\gamma \rightarrow \infty}\left|h_{\gamma}(t)\right|<\infty$ for almost all $t \in S$. From (14) we have for positive bounded functions $f$ and hence for all bounded functions $f$ that $\lim _{\gamma \rightarrow \infty} h_{\gamma}(t)=0, t \in S$. Let $\Lambda_{m}$ consist of all rational numbers $\gamma \geqq m$. For every $f \in L(S)$ the function $h_{\gamma}(t)$ is defined and continuous in $\gamma$ for almost all $t$ and hence, for every $f \in L(S), \sup _{\gamma} \in \Lambda_{m} h_{\gamma}(t)$ $=\sup _{\gamma \geq m} h_{\gamma}(t)$ and $\inf _{\gamma \in \Lambda_{m}} h_{\gamma}(t)=\inf { }_{\gamma \geq m} h_{\gamma}(t)$ for almost all $t \in S$. The desired conclusion then follows from Lemma 7 .

LEMMA 20. If $g \in L(S)$ and $T_{\gamma} g=g$ for every $\gamma>0$ then there is a null set $S_{0} \subset S$ such that $g_{\gamma}(t)=g(t), \gamma>0, t \in S-S_{0}$.

By hypothesis there is for each $\gamma>0$ a null set $S(\gamma)$ such that $g_{\gamma}(t)=g(t)$, $t \in S-S(\gamma)$. Let $S_{0}=\sum S(\gamma)$ where the sum is taken over all rational $\gamma$. Then $g_{\gamma}(t)=g(t)$ for $t \in S-S_{0}$ and all rational $\gamma$. The desired conclusion follows from the fact that $g_{\gamma}(t)$ is continuous in $\gamma$ for every $t$ where it is defined.

LEMMA 21. Statement (11) implies statement (13).

It is seen from (15), Lemma 19, and Lemma 20 that for every $f$ in a dense set in $L(S)$ (that is, for $f$ in $\mathfrak{M}+\mathfrak{N}$ ) the $\lim _{\gamma} f_{\gamma}(t)$ exists almost everywhere. Also for every $f \in L(S)$ (Lemma 18) we have $\lim _{\sup _{\gamma \rightarrow \infty}}\left|f_{\gamma}(t)\right|<\infty$ for almost all $t \in S$. Thus by taking $\Lambda_{m}$ in Lemma 7 to be the rational $\gamma \geqq m$ we have for every $f \in L(S)$

$$
\lim _{m} \sup _{\gamma \in \Delta_{m}} f_{\gamma}(t)=\lim _{m} \inf _{\gamma \in \Delta_{m}} f_{\gamma}(t),
$$

for almost all $t$. Since $f_{\gamma}(t)$ is continuous in $\gamma$ for every $t$ where it is defined as a Lebesgue integral the sup and inf appearing above may be taken over all $\gamma \geqq m$. This proves the lemma.

Lemma 14 and Lemma 21 together prove Theorem 2.

YALE UNIVERSITY, New Haven, Conn.

The Institute for Advanced Study, Princeton, N. J. 